\title{
Attributes of Hypoxic Preconditioning Determine the Complicating Atherogenesis of Plaques
}

\author{
Lawrence M Agius \\ Additional information is available at the end of the chapter \\ http://dx.doi.org/10.5772/51127
}

\section{Introduction}

Atherogenesis constitutes a prominent mechanism in inducing stenosis of the vascular lumen by multiple mechanisms within contextual reference of heterogeneity of pathways and phenotype determinants. Reduction of low-density lipoprotein-C has well-established value and constitutes a major guideline for cardiovascular disease prevention [38]. Redox state imbalance plays a role in preclinical atherosclerosis [7]. The realization of events constituting the acute coronary syndromes is particularly critical in the evolution of lesions that further compromise blood supply to target tissues. It is highly significant to consider the distribution of individual lesions with reference to disturbed blood flow patterns within the vascular arterial tree, as further evidenced by selectivity to vascular branch points. Disturbed flow may hinder transport of nitric oxide particularly distal to a stenosis [17].

Evidential parameters confirm a primarily quantitative series of dimensional effects that participates in the development of lesions and that ranges from constitutional progression to environmental gene promotion particularly in the activation and dysfunction of endothelial cells.

High density lipoprotein has a wide range of functions including antiatherogenic, anti-inflammatory and anti-oxidant action [30].

In such manner, also a highly heterogeneous series of conditioning influences participate in inducing not only the creation of individual atherosclerotic plaques but also the evolution of such plaques to unstable lesions inducing acute coronary events. Molecular mediators include members of the chemokine family of leukocyte chemoattractants and their G proteincoupled receptors [37]. 
Distributional patterns of lesion infliction correlate also with procoagulant effects that systemically compromise recoverability from injury to various components of the vascular wall.

\section{Patterned autonomy}

Patterned autonomy of lesion creation and of progression contrasts with a realization of promoted endothelial dysfunction in terms of quantitative dimensions. Lipids in atherosclerotic lesions weaken cellular antioxidant action through generation of $\mathrm{H}_{2} \mathrm{O}$ and promote plaque progression [33]. Lipoprotein plays a role in inducing endothelial dysfunction [32]. It is critically significant to view the distribution of lesions that arise as hemodynamic forces of laminar flow on the one hand and as disturbed dynamics of flow at vascular branch points.

The further participation of pathways of identifiable injury arise from a realization of ongoing progression of individual lesions that conform to tunica intimal targeting in lipoprotein deposition. Apolipoprotein E4 causes macrophage dysfunction and enhances apoptosis by inducing ER stress; it is a major genetic risk factor in atherosclerosis and diabetes [2].

The various component remodellings within the vascular intima are paramount consideration in the realization of an injury that goes beyond the concept of a primary endothelial form of injury. In such manner, the roles played by oxidized lipoproteins are central to a wide distributional series of patterns that are distinguished primarily by their quantitative attributes. Inflammation and metabolism are important drivers of atherogenesis in the context of HIV infection [18].

Primary disorders such as diabetes mellitus, hypertension, and abnormal homocysteine metabolism are examples of promoting pathways that contribute in the identification of susceptibility patterns of non-resolution of emerging atherosclerotic lesions in various loci within the arterial vascular tree. In such manner, compounding influences of highly heterogeneous nature constitute a specific marker in the pathogenesis of atherosclerosis. Inflammation and immunity in the "infection hypothesis" may form a biologic substrate for atherogenesis [28]. Fibroblast growth factor receptor 4 is implicated in vascular smooth muscle cell proliferation and atherosclerosis [5].

\section{Agonists}

Significant performance of injurious agonists allow for permissive emergence of dysfunctional endothelial cells in a mode of participation that includes a shift especially of phenotypic determination of such vascular wall components as smooth muscle cells from the tunica media. Within such a setting, the distributional attributes of a contractile versus secretory phenotype of smooth muscle cells allows for the expression of injurious agonists that further compromise the recoverability from endothelial cell injury in particular. The function of the ubiquitin-proteasome system deviates from the norm in atherogenesis and this 
may necessitate new UPS-based therapeutic modalities [29]. Indeed, the very identity of the dysfunctional state of overlying endothelial cells may prove a derived parameter of consequence within systems of active remodelling of the intima as induced by such phenotypic shifts in activity of smooth muscle cells in particular.

Aldose reductase in the polyol pathway promotes excessive accumulation of intracellular reactive oxygen species in various tissues of diabetic patients [34].

\section{Injury}

The systems of promotional realization of injury as induced by atherosclerotic plaques are a significant compound system that incriminates adhesion of monocytes to dysfunctional or activated endothelium.

The participation of injury to the arterial wall is complex and acts as a series of overlapping influences that further contributes to injury as evidenced by the action of evolving hypoxic influence and by procoagulant activity. Almost all coagulant proteins including tissue factor are found in atherosclerotic plaques [19]. Low matrix metalloproteinase-2 levels correlate with intra-cranial location of atherosclerosis [15].

The overlapping series of dynamic events in atherogenesis is permissive in promoting a pathway realization that is central to hypoxia inducing further progression of the lesions. High glycemic load glycemic index are related to significantly increased risk for atherogenesis in women in particular [20].

\section{Progression}

In such manner, the promotional distributional significance of concurrent foci of injury is paramount parameter in inducing the characterization of lesions that essentially progress. Toll-like receptor signalling may link chronic inflammation with cardiovascular disease progression and immune activation [31].

It is significant to view the parameters of quantitative nature in the development of individual lesions that hemodynamically are closely related often to disturbed blood flow at vascular branch points.

It is with referential background components of various identifiable elements of the vascular wall that atherogenesis proves an integrative phenomenon of progression in its own right. High density lipoprotein particle functionality is at least as important as HDL-C levels due to effects on inflammation, hemostasis and apoptosis [22].

The individual participating roles played by such processes as monocyte rolling and subsequent firm adhesion to endothelial cells helps characterize specific attributes of the activated or dysfunctional endothelium. The decreased production of nitric oxide by dysfunctional 
endothelium is a prototype example of distributional nature in denoting a systemic participation of further ongoing transformation in phenotype characterization of cellular components of the vascular wall as integrative phenomenon.

The realization of oxidized lipoproteins may well prove a central participant in the orchestration of events inducing injury as a self-progressive culmination in atherogenesis and in further progression of individual atherosclerotic plaques. Identification of pathway events that distribute the lesions within systems of a primarily promotional nature indicates that macrophages and foam cells promote atherogenesis as a primarily distributional series of quantitative nature. Epigenetic modification of the genome may link environmental injury to gene regulation [41]. Apoptosis and suppressed clearance of apoptotic macrophages render plaques susceptible to rupture, promoting thrombosis [13].

\section{Complicated plaque}

The complicated atherosclerotic plaque is thus a series of overlying pathways of influence that concurrently participate in identifying the different component systems in pathogenesis. Regulating $\mathrm{T}$ cells and serum interleukin-10 may exert a protective role against plaque rupture in patients with coronary atherosclerosis [11]. It is with contextual reference to oxidized lipoprotein deposits within the intima that phenomena of adherence to dysfunctional endothelium induce leukocytes as systemic agonists in atherogenesis. The role of platelets in atherothrombosis is well established [9].

The sharp distinction in identification of progression of an individual plaque from the ruptured plaque permits the emergence of multiple profiles in developmental history of lesions that individually evolve but that are systemically compounding and overlapping in profile determination. NF-E2 related factor 2 pathway restores redox homeostasis and Nrf2 cross talks with the proteasome [4].

The realization of injury to endothelial cells is therefore only an initial event in the once-realized reactivity to injury to multiple components of the vascular wall.

The dynamics of orchestration of various injurious agonists thus emerge as an essential component system in atherogenesis in a manner that calls into operative participation multiple heterogeneous pathways ranging from procoagulant effect of disturbed blood flow, hypoxia, dysfunctional reduction in nitric oxide production and action, and especially the chemotactic influences as induced by oxidized lipoproteins deposited in the intima. Arachidonic acid increases inflammation and enhances the ability of endothelial cells to bind monocytes in vivo [12]. The further participation of remodelling of the intima as a result of migration and proliferation of smooth muscle cells is evidence for a series of phenotypic switches that allow permissive injury to multiple cell components and to matrix production of proteoglycans.

It is within a systemically integrative series of active realizations that atherogenesis proves an integrative expression of component pathways; this paradoxically determines a com- 
pound pathobiologic profile that is individually determined by constituent components of the vascular wall affected. Macrophages are exquisitely sensitive to their microenvironment, influencing plaque rupture and thrombosis [40].

It is therefore in terms of quantitative realization that atherogenesis is both initiating and progressive influence in the determination of profile progression of the individual atherosclerotic plaque.

\section{Foam cells}

Foam cells are pivotal in inducing a series of chemotactic phenomena in atherogenesis in a manner that contributes to the self-progressive nature of the disease process. In terms of distributional injury, the paramount characterization of the processes in atherosclerosis is focused clinically in the emergence of the complicated atherosclerotic plaque. In such a setting, the contributions by procoagulation of the disturbed blood flow prove a central player in the determination of stenosis as predisposition to plaque rupture.

Attributing significant paramount dynamics in atherogenesis to a series of events of accumulative effect of oxidized lipoprotein is a characterization in the establishment of self-promotional progression within any individual atherosclerotic lesion. It is the realization of quantitative identification of such individual plaques that allows for the emergence of systemic effect within much of the vascular arterial tree.

Chronic inflammation is implicated in atherogenesis with cytokine involvement in all stages of plaque development [3].

The distributional dynamics of promotional events are primarily permissive in a mode of further contributing influence in atherogenesis. Hypoxia-inducible factor- 1 initiates formation of foam cells, endothelial cell dysfunction, apoptosis angiogenesis and progressive inflammation [10].

It is only in terms of a systemic event that integrates as the individual atherosclerotic plaque that one can realize a transformation of a primarily accumulative lesion to the complicated atherosclerotic plaque.

\section{Tunica intima}

The multi-component history of injury would account for a concordance influence in determining the realization of initiating injury to the endothelium and to the distributional contributions for further different forms of injury to other components of the vascular wall.

Parameters of progression are differential contributors to the essential nature of atherogenesis that is both dysfunctional and activating to such cell components as the endothelium. Estrogens have potent antioxidant activity and reverse endoplasmic reticulum stress in 
endothelial cells [14]. The proximity of the tunica intima to both overlying endothelium and to tunica media promotes the interactivity of smooth muscle cells within paracrine and autocrine systems of determining pathobiology. The significance attributed to such pathways as deposition of proteoglycan matrix within the intima proves a heterogeneity of involvement as significant characterization of multiple forms of progression of the individual atherosclerotic plaque. In such manner, distributional dynamics within foci of involvement by atherosclerosis allow for the emergence of parameters of progression that identifiably further promote permissive conditions of a quantitative nature in accumulation and chemotaxis of monocytes in particular.

\section{Nonresolution}

The complicated atherosclerotic plaque shows an essential neovascular component at its base and this appears to be a primary source for the establishment of complications such as intra-plaque hemorrhage and for rupture of the lipid core of the plaque into the vascular lumen. It is in terms of ongoing proliferation of a phenotypically secretory smooth muscle cell population that enlargement of the individual plaque proves self-progressive in dimensions and also self-progressive in terms of transforming dynamics to the complicated or ruptured plaque. In such manner, the overall contribution of injury to the overlying endothelium confirms dimensions of non-resolution beyond the fatty streak stage. Inflammasomes regulate proinflammatory caspases and interleukin-1 cytokines in response to various stimuli [24].

Integral participation of multiple foci of injury to a given point in vascular wall atherogenesis hence proves an inbuilt progression that is quantitatively determined but that allows a permissive microenvironment to promote transformability to the unstable plaque. It appears significant to view the unstable plaque as a transforming event in its own right beyond the dimensions of any individual atherosclerotic lesion. It is in such a setting that neovascularization of the plaque is a central agonist in the creation of acute coronary events as seen clinically.

The proinflammatory attributes of the atherosclerotic plaque accompany the dynamics of the neovascularization phenomenon in promoting the emergence of a permissive micro-environment within the vascular wall. Macrophages phagocytose apoptotic cells, clear necrotic debris and repair tissues; these are challenged by local cell stressors that include hypoxia, oxidative stress and protease activity [36].

The intimal remodelling is, in part, an expression of such pro-inflammatory activity. Immune responses to plaque antigens modulate inflammatory responses in the intima [39]. It is in terms of ongoing participation of new agonists that the atherogenesis proves a promotional agonist in its own right in determining the dynamics of vascular stenosis and also of instability as plaque rupture.

Thrombosis overlying the atherosclerotic plaque is a phenomenon as complicated plaque emergence and is believed to be a direct contributor to the establishment of further complications in clinically unstable angina. Venous and arterial thromboses are probably associat- 
ed with overlapping risk factors [8]. The overall dimensions of plaque evolution are hence highly complex, and such complexity is largely attributable to participants from the overlying luminally disturbed blood flow.

In spite of such considerations an essential role for hypoxia specifically affects the endothelium and other components of the vascular wall.

\section{Neovascularization}

The neovascularization at the base of the complicated atherosclerotic plaque may be an expression of such overall effects of hypoxia that transforms accumulative phenomena of atherogenesis as complicated plaques that rupture into the lumen.

The pro-inflammatory nature of plaques appears also expressive parameter of such hypoxia as a result of a neovascularity that further emerges as an over-riding phenomenon of permissiveness in atherogenesis. In this regard Nuclear Factor-kappaB plays an orchestrating role in formulating multiple heterogeneous elements in evolutionary permissiveness. As HIV infected patients age, atherosclerosis has become an increasing cause of morbidity and mortality, initiating immune and inflammatory responses [23].

The directional promotion in development of the unstable plaque is therefore an expression of transformational dynamics that promotes the rupture of the overlying fibrous cap and the extrusion of the lipid core. The biophysics of such fibrous cap appears instrumental particularly in the disruption of the junctional elements with the adjacent vascular wall.

The interplay of genetic factors with micro-environmental agonists is particularly significant in terms of the dynamics of lipid accumulation within the plaque [16].

Such phenomenal increments play contributing roles of the overlying blood flow in redistributing lipoproteins and cholesterol within the vascular wall. The dimensions of the lipid core are themselves determining agonists in plaque rupture, and a high content of such lipid core to over $40 \%$ of the overall plaque is significant in this regard.

Redistribution of attributes within the individual atherosclerotic plaque appears a promotional feature as pro-inflammatory effects and as plaque neovasculature. The hypoxic environment would account for permissive emergence of multi-component parameters that coordinate the characterization of the final complicated atherosclerotic plaque within dimensions of accumulation of lipid and transformation to plaque rupture.

\section{Integral atherogenesis}

The complicated atherosclerotic plaque as integral atherogenesis is an overall principal participation in the progression of a lesion that is both pro-inflammatory and enlarging. Advanced glycation end-products are implicated in the pathogenesis of diabetes-associated 
atherosclerosis by increasing smooth muscle cell susceptibility to insulin-like growth factor-1 mitogenic effects [6].

The foam cells within the plaque are an expression of integrative participation within such schemes of complicating plaque formation as realized by such phenomena as lipid core accumulation, chemotaxis, paracrine secretion of growth factors and cytokines and the expressive effects of oxidized lipoproteins in particular. The distributional nature of disturbed blood flow is significant in the role of selective participation of injury to the overlying endothelial cells that become permeable to the inflow of lipoproteins within the vascular wall. Low density lipoproteins play a major role in initiating progressive atherosclerosis whereas high density lipoproteins suppress inflammation and thrombosis [1].

Hence, an overall series of dimensional agonists conform to and further establish the emergence of hypoxic influence in the quantitative formulation of the individual atherosclerotic plaque within a highly permissive micro-environment of vascular wall pathology. The significance of multi-component participation is symptomatic of the essential activation of endothelial cells that are at the interface with disturbed blood flow. Also, the accelerated or aggressive forms of atherogenesis seen in many forms of dyslipoproteinemias illustrate such phenomenon within parametric contexts of endothelial cell dysfunction.

\section{Self-progression}

Directional proportions in redistribution of injury to the vascular wall are conceptually a mechanism of a quantitatively self-progressive establishment in atherogenesis.

The murine models of atherosclerosis, particularly the transgenic models of absent ApoE gene and of absent low density lipoprotein receptor fed on a Western diet indicate the role played by lipoproteins as initiators and promoters of an essentially permissive micro-environment that is instrumental in atherogenesis as micro-environmental conditioning and preconditioning. In such manner, conditional remodelling of microenvironmental factors involves the characterization of many of the contributing agonists in atherogenesis. Hypoxia of the endothelium and intima is a central theme in such conditioning and allows for the multiple agonists in atherogenesis to contribute to the essential individualization of the plaque within systemizing schemes of vascular wall atherosclerosis. Pathological angiogenesis enhances disease progression, increases macrophage infiltration and perpetuates necrosis and hypoxia [25].

Cellular phenotype switches are a feature affecting particularly endothelial expression of adhesion molecules and the generation of secretory roles for smooth muscle cells. P-selectins and to a lesser extent E-selectins are significant participants in adhesion of leukocytes to particular sites in the endothelium and allow for contributions also by vascular adhesion molecules and intercellular adhesion molecules and integrins. The matrix proteoglycans significantly accumulate as a result of such phenomena that promote chemotaxis of leukocytes from flowing blood and the drawing of smooth muscle cells into the intima. 
In such manner, distributional effects integrate as quantitative formulations of an atherosclerotic plaque that re-characterizes dimensions of phenotype switching and formulates roles for transforming cell types. Various nuclear receptors contribute to macrophage cholesterol metabolism, which in turn keeps the arteries in a chronically inflamed state [26].

\section{Endothelial dysfunction}

Incremental involvement of the intima corresponds to a progression that spatially conforms to effects of a disturbed blood flow that interacts with the endothelium. Dysfunctionality of endothelial cells arises within contexts of such interface phenomena in the realization of the quantitative attributes of increasing hypoxic injury and as dictated by the an accumulation of oxidized lipoprotein core.

Developmental parallels of involvement permit the destruction of the vascular intima that however promotes dimensions of aggregation of monocytes and the transformation to foam cells. In such manner, parameters of increasing involvement of the intima with atrophy of the tunica media progressively increases the abnormal flow of blood and promotes disturbed reactivity of endothelial cells. It is such cyclical disturbance that increases also the susceptibility to progressive accumulation of lipoprotein within the vascular wall.

A conceptual realization of developmental events is suggestive of a series of parallel pathways that coincidentally progress as overlapping systems of attempted reconstitution of pathways of possible recovery in the face of incremental destruction of the vascular wall. Monocyte recruitment into the vessel wall is a rate-limiting step in atherogenesis with a critical role played by reactive oxygen species [35].

\section{Permissiveness}

In such events promotional permissiveness would prove a determining series of further progression that calls into operative persistence the effects of hypoxia and of disturbed blood flow that primarily target the endothelial cell bed.

Extension, both laterally and also deeply into the vascular wall, requires the activation of response elements such as the shear stress response element that augments the distributional disturbance of interaction of tonicity of the vascular wall with disturbed dynamics of blood flow.

This phenomenon appears to augment further susceptibility to pro-inflammatory reactivity in the wake of an enlarging plaque that increasingly constitutes the phenotypic switching of such cell types as smooth muscle cells. Also, remodelling of the intima is both accumulative of proteoglycans and also modulatory in adapting to new blood flow dynamics.

Substantial reconstitution of the vessel wall, hence, is attempted in the form of replacement dynamics in the vascular wall in a manner that attempts the normalization, to some extent, 
of dynamics of blood flow. The adhesive molecules that are increasingly expressed on activated and dysfunctional endothelial cells allow for the ingress of leukocytes such as monocytes and memory $\mathrm{T}$ lymphocytes in the face of destruction of the vascular wall. Such parametric phenomena are paralleled by the procoagulant pathways that create and deposit thrombus on the surface of the complicated or ulcerated atherosclerotic plaque.

In such manner of progression, primary systems of permissiveness implicate a plasticity that is morphologically mirrored in dynamics of involvement of the plaque by the neovasculature invading the base of the plaque. It is in terms of ongoing incremental dynamics that the essential morphological and dysfunctional attributes of a modelled plaque lesion come to reconstitute a focal lesion of dimensional origin within the vascular intima but that eventually progresses as luminal stenosis and plaque rupture.

The multiplicity of plaque creation in the vascular intima denotes an ongoing cooperative series of disturbances emanating from significant exposure of the endothelium to such lesional promotional events as hypoxia and as further derived phenomena of a disturbed blood flow pattern. In such manner, the incremental mirroring of patterned progression in multiple plaques would correspond to representative further permissiveness in the face of increasing destruction of the vascular wall. High density lipoprotein reverses cholesterol transport and normalizes vascular function, in addition to antioxidative anti-inflammatory and anti-apoptotic actions [21].

Developmental aggregation of events hence is formulated as eventually complicated plaques that formulate thrombogenesis and deposition on the ulcerative but enlarging or complicated plaque.

Simple realization of events in plaque rupture comes to constitute a patterned progression that is transforming and which allows for the plasticity of reconstitutive pathways to emerge as complications of the atherosclerotic plaque.

It is perhaps in terms of significant interplay of multiple attempts at removal of oxidized lipoprotein that the dimensions of attempted reconstitution of the vascular wall come to operatively include a pro-inflammatory component in further promoting normalization of blood flow dynamics.

\section{Thrombosis}

The thrombus that is deposited on the surface of plaques is also an attempt at streamlining the luminal contours of the vessel wall in an attempt to accommodate new flow dynamics.

The response to injury hypothesis only partly accounts for the pathogenesis of an atherosclerotic plaque that is centered on a core of involvement of the vascular intima. It is the additional formulation of a hypoxic micro-environment that further proves a driving initiative in the development of serial plastic events that conformationally confirm the dimensions of a plaque reconstitution at the interface of abnormal blood flow dynamics. Hence, the redis- 
tributional series of phenomena come to model the individual lesion as hypoxic conditioning of the micro-environment and of attempts at neovascularization at the base of the lesion.

Developmental dynamics hence are paramount considerations in the evaluation of a specific plaque dimensionality. The overall conformations of lesions are centrally operative but dynamically progressive in the face of both accumulative and transforming pathways of agonist action and response adaptations. Hyperinsulinemia appears to promote macrophage foam cell formation and may thus promote atherogenesis in type-2 diabetics [27].

\section{Hypoxia}

Hypoxia is generated as a primary abnormality of the endothelium and as further propagated via the vasa vasorum supplying the vascular wall.

The parameters of further development of the injury to the endothelium allow for interface dynamics with abnormal blood flow that generate a secondary wave of proportional amplitude within the enlarging atherosclerotic plaque that evolves primarily as an end-stage complicated plaque lesion.

Distributional forces are driven by a series of hypoxic preconditions that promotes the characterization of individual lesions within context of further lesion infliction in other regions of the endothelium. It is such premise that accounts for a multiplicity of events that create a multifocal representation of atherosclerosis within the arterial vascular system, as denoted by parameters of blood flow dynamics and as hypoxia-driven plastic effects on the endothelium.

It is in such manner that paramount representative pathways conform to a response to injury and also to primary agonistic action of a hypoxic conditioning of the microenvironment of both endothelium and vascular intima.

Serial insult modulation of pathways include the re-establishment of multiple injuries to the endothelium that are essentially hypoxic in origin and which conform to the development of the plaque as primary emergent form of adaptation to endothelial involvement in particular.

\section{Concluding remarks}

The distributional anatomy of the individual atheromatous plaque is consistently reproduced in multiple regions of the arterial vascular tree and in a manner of conformational re-establishment of lesions as hypoxia of the endothelium and as dynamics of abnormal blood flow. In such manner, the constituent representations of micro-environment pre-conditioning is paramount driving force in the creation of an essential plaque that conformationally further propagates as multiple other plaques. The individuality of the plastic events in atherogenesis relate to vascular wall injury and to destruction within the contextual further evolution of lipoprotein core formation and as a series of potential complications. 
The neovascularization events are responsive elements to a hypoxia generated within the intima and affecting in particular the endothelial cells. Such endothelium is both activated to express adhesion molecules and also dysfunctional with particular reduction in nitric oxide production.

The further amplification of vascular wall injury is indicative of parameters of parallel but overlapping proportions in the creation of a highly plastic series of preconditioned microenvironments. It is in terms not only of a response to injury but also of a series of reactivities to hypoxia to the endothelium and intima that atherosclerosis proves a self-progressive lesion.

The dynamics of abnormal blood flow are constituent parameters to which the emergent plaque conforms to in partial manner. The neovascularization of the lesion core adopts the contextual conformation that responds to hypoxia and evolving enlargement of the individual atherosclerotic plaque.

\section{Author details}

Lawrence M Agius*

Address all correspondence to: lawrence.agius@um.edu.mt

Department of Pathology, Mater Dei Hospital, University of Malta Medical School, Malta

\section{References}

[1] Badimon, L., \& Viluhur, G. (2012). LDL-cholesterol versus HDL-cholesterol in the atherosclerotic plaque: inflammatory resolution versus thrombotic chaos. Ann NY Acad Sci Apr, 1254(1), 18-32.

[2] Cash, J. G., Basford, J. E., Jaeschke, A., Chatterjee, T. K., Weintraub, N. L., \& Hui, D. Y. (2012). Apolipoprotein E4 impairs macrophage efferocytosis and potentiates apoptosis by accelerating Endoplasmic Reticulum stress. J Biol Chem Jun 23.

[3] Catana, C. S., Cristea, V., Miron, N., \& Neagol, I. B. (2011). Is interleukin-17 a proatherogenic biomarker? Roum Arch Microbiol Immunol Jul-Sep , 70(3), 124-8.

[4] Chapple, S. J., Slowand, R. C., \& Mann, G. E. (2012). Crosstalk between Nrf2 and the proteasome : therapeutic potential of Nrf2 inducers in vascular disease and aging. Int J Biochem Cell biol May7.

[5] Chen, H., Tong, J., Zou, T., shi, H., Liu, J., Du, X., et al. (2012). Fibroblastgrowth factor receptor 4 polymorphisms are associated with coronary artery disease. Genet Test Mol Biomarkers May15. 
[6] Correra-Giannella, M. L., Andrade de Azevedo, M. R., Leroith, D., \& Giannella-Neto, D. (2012). Fibronectin glycation increases IGF-1 induced roliferation of human aortic smooth muscle cells. Diabetol Metab Syndr May 3 , 4(1), 19.

[7] De Chiara, B., Sedda, v., Parolini, M., Campolo, J., De Maria, R., Caruso, R., et al. (2012). Plasma total cysteine and cardiovascular risk burden: action and interaction. Scientific World Journal, 303654.

[8] Franchini, M., \& Mannucci, P. M. (2012, Jun). Association between venous and arterial thrombosis: clinical implications. Eur J Intern Med, 23(4), 333-7.

[9] Freynhofer, M. K., Bruno, V., Wojta, J., \& Huber, K. (2012). The role of platelets in Athero-thrombotic events. Curr Pharm Des Jun 19.

[10] Gao, L., Chen, Q., Zhou, X., \& Fan, L. (2012). The role of hypoxia-inducible factor 1 in atherosclerosis. J Clin Pathol May 8.

[11] George, J., Schwartzenberg, S., Medvedovsky, D., Jonas, M., Charach, G., Afek, A., \& Shamiss, A. (2012). Regulatory T cells and IL-10 levels are reduced in patients with vulnerable coronary plaques. Atherosclerosis Apr 6.

[12] Grenon, S. M., Aguado-Zuniga, J., Hatton, J. P., Owens, C. D., Conte, M. S., \& Hughes-Fuford, M. (2012). Effects of fatty acids on endothleial cells: inflammation and monocyte adhesion. J Surg Res apr 27.

[13] Gui, T., shimokado, A., Sun, Y., Akasaka, T., \& Muragaki, Y. (2012). Diverse roles of macrophages in atherosclerosis: from inflammatory biology to biomarker discovery. Mediators Inflamm, 693083.

[14] Hass, M. J., Raheja, P., Jaimungal, S., \& Sheikh-Ali, Mooradian. A. D. (2012). Estrogen-dependent inhibition of dextrose-induced endoplasmic reticulum stress and superoxide generation in endothleial cells. Free Radic Biol Med Apr18

[15] Jeon, S. B., Chun, S., Choi-Kwon, S., Chi, H. S., Nah, H. W., Kwon, S. U., et al. (2012). Biomarkers and location of atherosclerosis: Matrix metalloproteinase-2 may be related to intracranial atherosclerosis. Atherosclerosis Jun 8.

[16] Kovacic, S., \& Bakran, M. (2012). Genetic susceptibility to atherosclerosis. Stroke Res Treat, 362941.

[17] Liu, X., Fan, Y., Xu, X. Y., \& Deng, X. (2012). Nitric oxide transport in an axisymmetric stenosis. JR Soc Interface May 16.

[18] Lo, J., \& Plutzky, J. (2012, Jun). The biology of atherosclerosis: general paradigms and distinct pathogenic mechanisms among HIV-infected patients. J Infect Dis, 205(Suppl 3), S368-374.

[19] Loeffen, R., Spronk, H. M., \& Ten, Cate. H. (2012). The impact of blood coagulability on atherosclerosis and cardiovascular disease. J Thromb Haemost May 12 
[20] Ma, X. Y., Liu, J. P., \& Song, Z. Y. (2012). Glycemic load, glycemic index and risk of cardiovascular diseases: Meta-analyses of prospective studies. Atherosclerosis Jun 6.

[21] Mackness, B., \& Mackness, M. (2012). The antioxidant properties of high-density lipoporteins in atherosclerosis. Panminerva Md Jun, 54(2), 83-90.

[22] Mahdy, Ali. K., Wonnerth, A., Huber, K., \& Wojta, J. (2012). Cardiovascular disease risk reduction by raising HDL cholesterol-current therapies and future opportunities. Br J Pharmacol Jun 22.

[23] Maniar, A., Ellis, C., Asmuth, D., Pollard, R., \& Rutledge, J. (2012). HIV infection and atherosclerosis: evaluating the drivers of inflammation. Eur J Prev Cardiol May 3.

[24] Matsuura, E., Lopez, L. R., Shoenfeld, Y., \& Ames, P. R. (2012). Beta2glycoprotein 1 and oxidative inflammation in early atherogenesis: a progression from innate to adaptive immunity? Autoimm Rev Apr27.

[25] Moreno, P. R., Purushothaman, M., \& Purushothaman, K. R. (2012). Plaque neovascularization: defense mechanisms, betrayal, or a war in progress. Ann NY Acad Sci Apr; , 1254(1), 7-17.

[26] Nagy, Z. S., Czimmerer, Z., \& Nagy, L. (2012). Nuclear receptor mediated mechanisms of macrophage cholesterol metabolism. Mol Cell Endocrinol Apr 22.

[27] Park, Y. M., Kashyap, R. S., Major, J. A., \& Silverstein, R. L. (2012). Insulin promotes macrophage foam cell formation: potential implications in diabetes-related atherosclerosis. Lab invest Apr 23.

[28] Pedicino, D., Giglio, A. F., Galiffa, V. A., Claidella, P., Trotta, F., Graziani, F., et al. (2012). Infections, immunity and atherosclerosis: Pathogenic mechanisms and unsolved questions. Int J Cardiol Jun 22.

[29] Powell, S. R., Herrmann, J., Lermen, A., Patterson, C., \& Wang, X. (2012). The ubiquitin-proteasome system and cardiovascular disease. Prog Mol Biol Transl Sci, 109, 295-346.

[30] Soran, H., Hama, S., Yadav, R., \& Darrington, P. N. (2012). HDL Functionality. Curr Opin Lipidol Jun 22.

[31] Spirig, R., Tsui, J., \& Shaw, S. (2012). The emerging role of TLR and innate immunity in cardiovascular disease. Cardiol Res Pract, 181394.

[32] Stancu, C. S., Toma, L., \& Sima, A. V. (2012). Dual role of lipoproteins in endothelial cell dysfunction in atherosclerosis. Cell Tissue ResMay 18.

[33] Szuchman-Sapir, A., Etzman, M., \& Tamir, S. (2012). Human atherosclerotic plaque lipid extract impairs the antioxidant defense capacity of monocytes. Biochem Biophys Res Commun Jun 20.

[34] Tang, W. H., Martin, K. A., \& Hwa, J. (2012). Aldose reductase, oxidative stress, and diabetes mellitus. Front Pharmacol, 3, 87. 
[35] Tavakoli, s., \& Asmis, R. (2012). Reactive oxygen species and thiol redox signaling in the macrophage biology of atherosclerosis. Antioxid Redo Signal Apr 29.

[36] Thorpe, E. B. (2012). Contrasting inflammation resolution during atherosclerosis and post-mocardial infarction at the level of monocyte/macrophage phagocytic clearance. Front Immunol, 3, 39.

[37] Wan, W., \& Murphy, P. M. (2011). Regulation of atherogenesis by chemokine Receptor CCR6. Trends Cardiovasc Med Jul , 21(3), 140-144.

[38] Whayne, T. F., Jr. (2012). Assessment of low-density lipoprotein targets. Angiology Jun 25.

[39] Wigren, M., Nilsson, J., \& Kolbus, D. (2012). Lymphocytes in atherosclerosis clin. Chim Acta May4.

[40] Williams, H. J., Fisher, E. A., \& Greaves, D. R. (2012). Macrophage differentiation and function in atherosclerosis: opportunities for therapeutic intervention? J Innate Immun Apr 27.

[41] Xu, S. S., Alam, S., \& Margariti, A. (2012). Epigenetics in Vascular Disease-therapeutic potential of new agents. Curr Vasc Pharmacol Jun 22. 
